\title{
BMJ Open Effects of mechanical interventions in the management of knee osteoarthritis: protocol for an OA Trial Bank systematic review and individual participant data meta-analysis
}

Erin M Macri (D) , ${ }^{1,2}$ Michael Callaghan, ${ }^{3}$ Marienke van Middelkoop, ${ }^{2}$ Miriam Hattle, ${ }^{4}$ Sita M A Bierma-Zeinstra ${ }^{1,2}$

To cite: Macri EM, Callaghan M, van Middelkoop M, et al. Effects of mechanical interventions in the management of knee osteoarthritis: protocol for an OA Trial Bank systematic review and individual participant data meta-analysis. BMJ Open 2021;11:e043026. doi:10.1136/ bmjopen-2020-043026

- Prepublication history and additional materials for this paper is available online. To view these files, please visit the journal online (http://dx.doi org/10.1136/bmjopen-2020043026).

Received 22 July 2020 Revised 21 December 2020 Accepted 20 January 2021

Check for updates

(c) Author(s) (or their employer(s)) 2021. Re-use permitted under CC BY. Published by BMJ.

${ }^{1}$ Department of Orthopaedics and Sports Medicine, Erasmus University Medical Center, Rotterdam, The Netherlands ${ }^{2}$ Department of General Practice, Erasmus University Medical Center, Rotterdam, The Netherlands

${ }^{3}$ Department of Health

Professions, Manchester

Metropolitan University,

Manchester, UK

${ }^{4}$ School of Primary, Community and Social Care, Keele University, Keele, UK

Correspondence to

Dr Erin M Macri;

e.macri@erasmusmc.nl

\section{ABSTRACT}

Introduction Knee osteoarthritis $(O A)$ is a prevalent and disabling musculoskeletal condition. Biomechanical factors may play a key role in the aetiology of knee $0 A$, therefore, a broad class of interventions involves the application or wear of devices designed to mechanically support knees with $0 \mathrm{~A}$. These include gait aids, bracing, taping, orthotics and footwear. The literature regarding efficacy of mechanical interventions has been conflicting or inconclusive, and this may be because certain subgroups with knee $0 A$ respond better to mechanical interventions. Our primary aim is to identify subgroups with knee $0 A$ who respond favourably to mechanical interventions.

Methods and analysis We will conduct a systematic review to identify randomised clinical trials of any mechanical intervention for the treatment of knee $0 \mathrm{~A}$. We will invite lead authors of eligible studies to share individual participant data (IPD). We will perform an IPD meta-analysis for each type of mechanical intervention to evaluate efficacy, with our main outcome being pain. Where IPD are not available, this will be achieved using aggregate data. We will then evaluate five potential treatment effect modifiers using a two-stage approach. If data permit, we will also evaluate whether biomechanics mediate the effects of mechanical interventions on pain in knee $0 A$.

Ethics and dissemination No new data will be collected in this study. We will adhere to institutional, national and international regulations regarding the secure and confidential sharing of IPD, addressing ethics as indicated. We will disseminate findings via international conferences, open-source publication in peer-reviewed journals and summaries posted on websites serving the public and clinicians.

PROSPERO registration number CRD42020155466.

\section{INTRODUCTION}

Knee osteoarthritis (OA) is a chronic musculoskeletal condition that affects approximately $24 \%$ of older adults. ${ }^{1}$ It is associated with substantial pain, loss of function and reduced quality of life. ${ }^{2}$ There are currently

\section{Strengths and limitations of this study}

- We designed our protocol in collaboration with the osteoarthritis Trial Bank, an internationally recognised organisation with considerable individual participant data (IPD) experience, including established procedures for navigating the safe transfer and storage of IPD.

- IPD meta-analyses of randomised clinical trials enhance the ability to handle participant-level and study-level confounding, and increases the power to identify responder subgroups and mechanisms underlying treatment effects.

- A key limitation to undertaking IPD analyses relates to overcoming data-sharing hurdles, and the achievement of our aims will in part depend on the ability to successfully obtain IPD from eligible studies.

no known disease-modifying treatment approaches available for knee OA. Current guidelines recommend a core approach of exercise, education and dietary weight management if appropriate. ${ }^{3}$ Adjunct interventions recommended for symptom management include pharmaceuticals such as non-steroidal anti-inflammatories, but also certain non-pharmacological interventions such as gait aids. ${ }^{3}$ At the end stages of knee OA, when pain and disability become severe enough, knee arthroplasty is often undertaken. However, approximately $20 \%$ of individuals who undergo knee arthroplasty report not being satisfied following surgery. ${ }^{4-6}$ With no effective disease-modifying treatment options, individuals often spend decades living with pain and disability. ${ }^{7}$ It is thus clinically imperative to identify interventions that can contribute to symptom management in individuals living with knee OA. 
Biomechanical factors, such as bony malalignment or poor movement patterns, may play a key role in the aetiology of knee OA by contributing to abnormal forces across affected joints. ${ }^{8}$ Therefore, one broad class of interventions for knee OA involves application, or wear, of devices aimed at presumably improving an individual's biomechanics to reduce joint forces, improve symptoms and potentially modify the disease trajectory. Such interventions include gait aids such as canes, but also bracing, taping, orthotics and footwear, and they can easily serve as adjuncts to the current recommended core exercisefocused programmes. ${ }^{3} 910$ This may be particularly relevant in individuals with certain biomechanical anomalies—such as frontal plane knee malalignment—because these individuals may be less likely to respond favourably to exercise. ${ }^{11}$ These commonly prescribed treatments are relatively inexpensive, less invasive and have fewer side effects compared with other medical approaches such as intra-articular injections, oral medications or surgery. ${ }^{12-14}$ Systematic reviews suggest some such mechanical interventions (eg, knee braces) may improve pain, though results have been conflicting or inconclusive regarding other outcomes or in other mechanical interventions. ${ }^{12-21}$

One possible reason for conflicting results is that knee OA represents a heterogeneous disease and certain subgroups with knee OA may respond better to mechanical interventions than others. ${ }^{22-24}$ For example, lateral wedge insoles and insoles with subtalar strapping may be more effective in individuals with varus knee alignment, ${ }^{13141721}$ while medial wedge insoles may be more effective in individuals with valgus knee alignment. ${ }^{14}$ Including both of these groups of individuals in the same study could mask true treatment effects. While subgroup analyses within such a trial may successfully identify a 'responder' subgroup, most trials are not powered for such secondary analyses, and evaluating multiple possible subgroup characteristics further increases the likelihood of spurious findings ${ }^{25-27}$ Confirming the existence of such 'responder' subgroups could lead to identifying effective targeted biomechanical interventions in knee OA.

In addition to subgroup characteristics, another possible reason for conflicting results is the mechanism by which such interventions impart their effect. If the effect of mechanical interventions is mediated by a change in some biomechanical feature, then it may be that different types (eg, brace vs tape), design or dose of intervention will confer different outcomes via differing influence on the mediating feature. ${ }^{28}$ Confirming the mediating role that biomechanics may play in mechanical interventions could help to optimise the design and application of mechanical interventions.

Several systematic reviews have evaluated the efficacy of mechanical interventions in knee OA, however, very little attention has been given to treatment effect modifiers or mediation analyses. ${ }^{12-21}$ Moreover, to our knowledge, no study on this topic has yet to pool individual participant data (IPD) across studies. An IPD meta-analysis evaluates raw units of data rather than aggregate study-level data, and is thus a more robust approach to evaluating treatment effect modifiers and mediators. ${ }^{29}{ }^{30}$ Compared with traditional study-level meta-analyses, IPD meta-analyses of randomised controlled trials (RCTs) enhances the ability to handle participant-level and study-level confounding, and increases the power to identify responder subgroups and mechanisms underlying treatment effects. ${ }^{30}$ The results using such an approach may, therefore, be more reliable and generalisable. ${ }^{30}$

Despite the growing recognition of the ethical and scientific importance of data sharing and scientific transparency, one of the biggest challenges in undertaking IPD analyses relates to overcoming data-sharing hurdles. ${ }^{31-33}$ Barriers range from successfully reaching original study authors; willingness or ability of authors to share data; and international ethics and regulations issues. ${ }^{31-33}$ The OA Trial Bank is an internationally recognised organisation that was established in 2010 and has developed procedures for navigating these barriers, including safely sharing, handling and storing IPD data from RCTs. ${ }^{34}$ The OA Trial Bank steering committee supports and approves all projects, including providing input on research questions and study methods. This is, therefore, the ideal organisation in the field of clinical OA research to collaborate with in successfully conducting an IPD meta-analysis.

\section{AIMS}

We aim to conduct a systematic review and IPD metaanalysis of RCTs, under the guidance of the OA Trial Bank, to evaluate the efficacy of mechanical interventions (ie, bracing, taping, orthoses, footwear or canes) in managing knee OA symptoms. Our primary aim is to identify subgroups of individuals with knee OA who respond favourably to mechanical interventions. Our secondary aim is to evaluate the effect of biomechanics as a mediator between mechanical interventions and symptoms.

\section{METHODS AND ANALYSES}

The OA Trial Bank steering committee approved a summary of this study protocol prior to preparing the full study protocol, ${ }^{34}$ and we published a basic study protocol with the International Prospective Register of Systematic Reviews. ${ }^{35}$ The current detailed protocol has been written in accordance with the Preferred Reporting Items for Systematic Reviews and Meta-Analyses Protocols (PRISMA) ${ }^{36}{ }^{37}$ statement and PRISMA IPD (PRISMA-IPD) guidelines. ${ }^{29}$ In collaboration with the OA Trial Bank, we will use methods described previously to guide the transfer and use of IPD, ${ }^{38-40}$ updated recently to adhere to current European data-sharing regulations. ${ }^{41}$

We will search for relevant studies in five databases: MEDLINE, Embase, CINAHL, CENTRAL and Web of Science, with dates from inception to search date. An initial search was completed 21 August 2019, and we will update this search prior to beginning data analyses. We will develop a search strategy in collaboration with 
an Erasmus MC librarian, using key words and medical subject headers, and adapting the syntax to the respective indexing vocabularies of each database (see online supplemental appendix 1).

We will identify studies that meet the following eligibility criteria:

\section{Participants}

Adults (18 years or older) with knee OA (tibiofemoral or patellofemoral), diagnosed using any common method (eg, radiographs, MRI, clinical criteria, diagnosis by a healthcare professional). We will exclude studies where $\mathrm{OA}$ is determined by self-report alone. We will include post-traumatic OA, in particular knees with OA secondary to anterior cruciate ligament injury, regardless of whether or not they were previously reconstructed or repaired. We will, however, exclude knees with non-traumatic OA that have undergone major surgical procedures such as tibial osteotomy or total knee arthroplasty. We will exclude rheumatoid or other inflammatory arthropathies. If a study contains a subgroup of participants that meet our inclusion criteria, we will include that study if IPD data are retrieved, or if subgroup analyses are reported in the original publication.

\section{Interventions}

Any intervention involving use or wear of mechanical devices (eg, bracing, taping, orthotics, footwear, cane) that is evaluated after more than 1 day or application of wear/use. We will include studies that combine these interventions with exercise or education/advice.

\section{Comparison}

Any non-surgical treatment (eg, placebo, usual care, any other intervention that does not involve surgery), waiting list or no treatment.

\section{Outcomes}

Our main outcome will be pain at the end of the studyspecific primary duration of treatment. Treatment duration will be categorised as short term ( $<4$ weeks), medium term (4-12 weeks) and long term ( $>3$ months). Outcome measures will also be extracted at additional time points during treatment. We will not extract outcomes at any time points that are measured after discontinuation of treatment. Secondary outcomes will include function, quality of life, global perceived change, OA feature severity, biomechanics and adverse events.

\section{Study design}

We will include peer-reviewed RCTs (or quasi-RCTs). We will exclude any other study design (eg, non-RCTs, prestudy and poststudy designs, observational studies). We will also exclude RCTs that only measure the acute effects of a single application of treatment.

\section{Languages}

English, Dutch, German, French
Titles and abstracts will be initially imported into EndNote X9 (Clarivate Analytics) for deduplication, and then imported into Covidence for screening. ${ }^{42}$ Two independent reviewers (EMM and MC) will screen titles and abstracts of all studies identified through this search strategy. ${ }^{43} \mathrm{~A}$ third reviewer (MvM) will be consulted in the event of unresolved disagreements. Following the initial screening, two reviewers (EMM and MC) will independently review full text manuscripts to identify studies for inclusion in this review. A third reviewer (MvM) will again be consulted in the event of disagreements. We will review reference lists of included studies and relevant reviews for additional eligible studies.

For all included studies, we will contact the corresponding author by email. If a current email address cannot be found or the author does not respond (up to three attempts), we will attempt to reach them by other means (eg, phone, post, contact institution). Where IPD are available and authors or institutions are willing to share data, a data delivery agreement will be signed by both parties. Where local ethics regulations require it, ethics approval will be sought prior to sharing data. Pseudonymised or anonymised data sets (all formats are acceptable, eg, SPSS, Excel) and related data dictionaries will then be transferred and stored securely on a database at Erasmus MC, for use only as agreed on in the data delivery agreement. One original study investigator (first or senior author, at the discretion of the data owner) will be invited to be a coauthor of the project if they are willing to assume responsibilities that meet authorship guidelines.

We will convert all data sets to a common format, combine data sets with a new variable identifying original trial and harmonise variables. Data checking will include evaluating baseline characteristics and results of comparisons for our main outcomes against results reported in original publications. We will also check for balancing of baseline participant characteristics in each treatment arm, and evaluate the extent to which all randomised participants in the IPD datasets have been included in study analyses. Authors will be consulted in the case of any inconsistencies or discrepancies. In cases where discrepancies cannot be resolved, we will (on a case by case basis) either conduct a sensitivity analysis with that study removed, or we will exclude the study from our analysis altogether.

\section{Data extraction}

Two independent investigators will extract data from all included published studies. From each study, we will extract the following data: country of study; funding source; study design; sample size; target population; inclusion/exclusion criteria; participant characteristics (age, sex, BMI, history of injury or surgery, comorbidities, psychosocial profile, metabolic profile, physical activity/ fitness, lifestyle factors, medication use); type, dose and context of intervention (including compliance, cointerventions, protocol deviations, adverse events, drop-out 
or withdrawal details); OA characteristics (compartment involvement, prevalence, severity, tissues involved), including pre-post if available; and pain, function, quality of life and biomechanics (eg, proprioception, knee alignment, strength, kinematics, kinetics) prepost as available. Global perceived change will also be extracted as available. For all patient-reported outcomes, we will extract recall period in addition to the outcome. For all outcome measures, notably biomechanics, we will also extract whether scores and measures are taken with respect to the device applied/worn or removed. Where IPD are available, we will conduct all analyses using IPD instead of aggregate data, following data consistency checks described above.

\section{Risk of Bias}

Two coauthors will independently evaluate risk of bias (ROB) for each included study using the Cochrane ROB tool V.2, ${ }^{44}$ and disagreements will be resolved by a third investigator. The Cochrane ROB V.2 considers five domains of possible bias: randomisation; deviations from intended interventions; missing outcome data; measurement of the outcome and selection of the reported results. For each domain, ROB is rated as low, some concerns or high. Both the original publications and IPD datasets will be used for evaluating ROB. The overall study will be considered to be of low ROB if all five domains are rated as low ROB, and high overall ROB if at least one domain is rated as high $\mathrm{ROB}$ or if some concerns are identified in multiple domains. We will consult original authors in the event of inadequate reporting or inconsistencies.

\section{Statistical analyses}

We will conduct an IPD meta-analysis of short term $(<4$ weeks), medium term (4-12 weeks) and long term ( $>3$ months) effects of mechanical treatments (alone or in combination with exercise or education/advice) in comparison to other non-surgical treatments, sham, waitlist or usual care.

Where within-study missing data are sparse (less than $5 \%$ ), we will assume data are missing completely at random and we will conduct complete case analyses, given the trivial loss of power and negligible implications on bias. ${ }^{45}$ Where higher proportions of data are missing within a study, we will conduct within-study multiple imputation. ${ }^{45}{ }^{46}$ In cases where a variable was not collected in a given study, we will exclude that study from the relevant analyses.

\section{Treatment efficacy}

To evaluate treatment efficacy, we will employ a two-step meta-analysis, first analysing each trial separately, and then pooling results across trials. ${ }^{30} 3847$ In step 1, within each trial, we will evaluate the effect of assigned intervention by intention to treat, regardless of method used in the original study. We anticipate that our main outcome, pain, will be evaluated differently across studies. To navigate this, we will evaluate the pain-related outcome from each study that ranks highest on the recommended
Table 1 Hierarchy of pain-related outcomes proposed by Jüni et $a l^{48}$

\begin{tabular}{|c|c|}
\hline Rank & Pain outcome \\
\hline \multirow[t]{9}{*}{ Highest } & Global pain score (eg, NRS, VAS) \\
\hline & Pain on walking (same as one but task-specific) \\
\hline & WOMAC pain subscale ${ }^{59}$ \\
\hline & Other composite pain scores (eg, KOOS Pain) ${ }^{60}$ \\
\hline & $\begin{array}{l}\text { Pain on activities other than walking (eg, stair } \\
\text { climbing) }\end{array}$ \\
\hline & $\begin{array}{l}\text { WOMAC global score (all three subscales } \\
\text { combined) }\end{array}$ \\
\hline & Lequesne osteoarthritis index global score ${ }^{61}$ \\
\hline & Other algo-functional composite scores \\
\hline & Patient's global assessment \\
\hline Lowest & Physician's global assessment \\
\hline
\end{tabular}

KOOS, Knee injury and Osteoarthritis Outcome Score; NRS, Numeric Rating Score; VAS, Visual Analogue Scale; WOMAC, Western Ontario McMaster University Osteoarthritis Index.

hierarchy of pain-related outcomes to be used for metaanalyses $^{48}$ (see table 1). For each available time point (short term, medium term and long-term), we will fit an analysis of covariance (ANCOVA) model to obtain the treatment effect estimate, including baseline pain as a covariate. ${ }^{49}$ We will report effect sizes from the ANCOVA model and their respective $95 \%$ CI. If study heterogeneity prevents us from harmonising pain data, then we will navigate this using a statistical approach based on available data. This will likely involve transforming data into standardised means differences or applying proportion of maximum scaling methods. ${ }^{50}$

In studies where we are unable to obtain IPD, we will extract aggregate data from published manuscripts as they are reported, for example, based on final scores or change scores. ${ }^{51}$ Similar models will be performed for secondary outcomes as data permit. In cases of dichotomous outcomes, we will perform binary modelling and report effect sizes as relative risk (RR, 95\% CI).

In step 2, we will perform random effects meta-analysis employing restricted maximum likelihood. ${ }^{52}$ We will perform separate meta-analyses for each type of intervention (eg, braces, taping) ${ }^{30} 4753$ We will report study heterogeneity as $\mathrm{I}^{2}$ and $\tau^{2} .{ }^{5455}$ In cases of notable heterogeneity $\left(\mathrm{I}^{2}>50 \%\right),{ }^{55}$ we will consider possible sources such as device design, treatment duration, comparison treatment, treatment adherence or study quality. We will then consider performing meta-regression, subgroup analysis or sensitivity analyses to explain or account for these potential sources of heterogeneity. We will pool results of studies both with and without IPD data after verifying that effect sizes of IPD studies do not differ from non-IPD studies. ${ }^{30} 51$

In addition to a two-step meta-analysis, we will also perform a one-step meta-analysis as a sensitivity analysis. 
In a one-step meta-analysis, all IPD datasets are harmonised into one large dataset and analysed together, with the addition of a covariate indicating original trial.

Within studies for which we have IPD and that report adherence to treatment, we will evaluate correlations between adherence and treatment effects. Where IPD are not available, we will extract aggregate data if reported. While we expect clinical and statistical heterogeneity to prevent meaningful meta-analysis of these data, we will pool data where possible.

\section{Treatment effect-modifier analyses}

We will conduct treatment effect-modifier analyses to identify subgroups of individuals with knee $\mathrm{OA}$ who respond to various mechanical interventions. ${ }^{38}$ We have proposed several subgroup characteristics that we hypothesise may modify the effect of mechanical interventions on our main outcome, based on expert opinion. These proposed subgroups include the following baseline characteristics: (1) mild vs severe OA (more severe joint space narrowing is associated with joint malalignment which may respond differently to mechanical interventions); (2) location of OA, specifically tibiofemoral versus patellofemoral $\mathrm{OA}$, medial versus lateral tibiofemoral OA, or medial versus lateral patellofemoral OA (location of cartilage damage may be associated with differing joint alignment or source of symptoms); (3) varus versus valgus knee alignment (may be associated with different localised joint forces); (4) obese versus non-obese (may confer different amounts of mechanical stress) and (5) post-traumatic versus non-traumatic OA (possible different biomechanical anomalies). Where feasible, we will apply a two-stage approach, whereby we first investigate within-trial interactions within each study using IPD data, then pool results across trials. ${ }^{27} 53$ This approach separates within-trial variation from acrosstrial variation, thus reducing the risk of ecological bias by analysing the effect of interest for individuals rather than groups of individuals. ${ }^{27}$ We will conduct all treatment effectmodifier analyses in IPD data only. ${ }^{53}$

\section{Mediation analyses}

We hypothesise that biomechanical factors may mediate the effect of these interventions (eg, kinematics, kinetics, proprioception, hypermobility) by reducing or normalising joint forces, which in turn reduces pain. If possible, we will conduct mediation analyses to evaluate this hypothesis. ${ }^{38}$ We acknowledge that it is rare for studies to evaluate biomechanical variables both before-and-after treatment, so undertaking this analysis will depend on whether there are sufficient data available in included studies. If such an analysis is possible, we will employ a single mediator model to evaluate the proportion of the total effect of the intervention on pain that is mediated by a change in biomechanics. ${ }^{56}$

We will conduct funnel plot analyses where there are at least ten studies for a given intervention, to consider the possible effects of small sample size or publication bias. ${ }^{57}$ We will summarise the overall level of evidence for each category of intervention using the GRADE approach. ${ }^{58}$
All analyses will be performed using Stata V.15.1 (StataCorp).

\section{Patient and public involvement}

Patients and the public were not directly involved in the design of this study protocol. The OA Trial Bank advisory board includes patient members who provide overall input to the OA Trial Bank Steering Committee activities. We will solicit patient involvement through the OA Trial Bank advisory board and also through our institutional patient advisors (www.arthrosegezond.nl) for input in the analysis and interpretation of our study results, and to inform and guide dissemination of our study results.

\section{ETHICS AND DISSEMINATION}

No new data will be collected, so de novo ethics approval is not required for our study. The OA Trial Bank has established protocols in place to guide the confidential and secure transfer and use of pseudonymised IPD $^{38-40}$ that adheres to current European data-sharing regulations. ${ }^{41}$ We will collaborate with data deliverers to also adhere to relevant institutional, national or international regulations regarding data sharing and ethics. We will store all IPD datasets on a secure driver in accordance with OA Trial Bank procedures. We will disseminate findings via international conferences, open-source publication in peer-reviewed journals and summaries posted on websites serving the public and clinicians.

\section{Twitter Erin M Macri @erin_macri}

Acknowledgements We thank Dr. Wichor Bramer at Erasmus MC for assisting us with developing our search terms and managing our search.

Contributors The study was conceptualised by EMM, MvM and SMAB-Z. MvM and SMAB-Z provided specific guidance regarding OA Trial Bank procedures. Study design and methodology was performed by EMM, MC, MvM, MH, and SMAB-Z. Statistical expertise was provided by $\mathrm{MH}$. EMM led the draft of the manuscript, and $\mathrm{MC}, \mathrm{MvM}, \mathrm{MH}$, and SMAB-Z all provided substantial intellectually input to the manuscript. All authors approved the final manuscript.

Funding EMM was funded by a Canadian Institutes of Health Research (CIHR) Banting Postdoctoral Fellowship. The OA Trial Bank has received funding from ReumaNederland (grant number $\mathrm{ClO}-01$ ).

Competing interests SMAB-Z reports grants from The Netherlands Organisation for Health Research and Development, CZ, European Union, Foreum, Dutch Arthritis Association, personal fees from 0steoarthritis Research Society International (OARSI), personal fees from Infirst Healthcare, and personal fees from Pfizer, all outside of the submitted work. We have no other disclosures.

Patient consent for publication Not required.

Provenance and peer review Not commissioned; externally peer reviewed.

Supplemental material This content has been supplied by the author(s). It has not been vetted by BMJ Publishing Group Limited (BMJ) and may not have been peer-reviewed. Any opinions or recommendations discussed are solely those of the author(s) and are not endorsed by BMJ. BMJ disclaims all liability and responsibility arising from any reliance placed on the content. Where the content includes any translated material, BMJ does not warrant the accuracy and reliability of the translations (including but not limited to local regulations, clinical guidelines, terminology, drug names and drug dosages), and is not responsible for any error and/or omissions arising from translation and adaptation or otherwise.

Open access This is an open access article distributed in accordance with the Creative Commons Attribution 4.0 Unported (CC BY 4.0) license, which permits others to copy, redistribute, remix, transform and build upon this work for any purpose, provided the original work is properly cited, a link to the licence is given, 
and indication of whether changes were made. See: https://creativecommons.org/ licenses/by/4.0/.

ORCID iD

Erin M Macri http://orcid.org/0000-0003-2798-6052

\section{REFERENCES}

1 Pereira D, Peleteiro B, Araújo J, et al. The effect of osteoarthritis definition on prevalence and incidence estimates: a systematic review. Osteoarthritis Cartilage 2011;19:1270-85.

2 Guccione AA, Felson DT, Anderson JJ, et al. The effects of specific medical conditions on the functional limitations of elders in the Framingham study. Am J Public Health 1994;84:351-8.

3 Bannuru RR, Osani MC, Vaysbrot EE, et al. OARSI guidelines for the non-surgical management of knee, hip, and polyarticular osteoarthritis. Osteoarthritis Cartilage 2019;27:1578-89.

4 Beswick AD, Wylde V, Gooberman-Hill R, et al. What proportion of patients report long-term pain after total hip or knee replacement for osteoarthritis? A systematic review of prospective studies in unselected patients. BMJ Open 2012;2:e000435.

5 Hawker GA, Badley EM, Croxford R, et al. A population-based nested case-control study of the costs of hip and knee replacement surgery. Med Care 2009;47:732-41.

6 Robertsson O, Dunbar M, Pehrsson T, et al. Patient satisfaction after knee arthroplasty: a report on 27,372 knees operated on between 1981 and 1995 in Sweden. Acta Orthop Scand 2000;71:262-7.

7 Ackerman IN, Kemp JL, Crossley KM, et al. Hip and knee osteoarthritis affects younger people, too. J Orthop Sports Phys Ther 2017;47:67-79.

8 Felson DT. Osteoarthritis as a disease of mechanics. Osteoarthritis Cartilage 2013;21:10-15.

9 Crossley KM, Vicenzino B, Lentzos J, et al. Exercise, education, manual-therapy and taping compared to education for patellofemoral osteoarthritis: a blinded, randomised clinical trial. Osteoarthritis Cartilage 2015;23:1457-64.

10 Skou ST, Roos EM, Laursen MB, et al. Total knee replacement and non-surgical treatment of knee osteoarthritis: 2-year outcome from two parallel randomized controlled trials. Osteoarthritis Cartilage 2018;26:1170-80.

11 Quicke JG, Runhaar J, van der Windt D, et al. Moderators of the effects of exercise for hip and knee osteoarthritis: a systematic review of subgroup analyses from randomised controlled trials. Osteoarthritis Cartilage 2020;28:S161.

12 Gohal C, Shanmugaraj A, Tate P, et al. Effectiveness of valgus offloading knee braces in the treatment of medial compartment knee osteoarthritis: a systematic review. Sports Health 2018;10:500-14.

13 Penny P, Geere J, Smith TO. A systematic review investigating the efficacy of laterally wedged insoles for medial knee osteoarthritis. Rheumatol Int 2013;33:2529-38.

14 Raja K, Dewan N. Efficacy of knee braces and foot orthoses in conservative management of knee osteoarthritis: a systematic review. Am J Phys Med Rehabil 2011;90:247-62.

15 Mine K, Nakayama T, Milanese S, et al. The effectiveness of braces and orthoses for patients with knee osteoarthritis: a systematic review of Japanese-language randomised controlled trials. Prosthet Orthot Int 2017;41:115-26.

16 Cherian JJ, Jauregui JJ, Leichliter AK, et al. The effects of various physical non-operative modalities on the pain in osteoarthritis of the knee. Bone Joint J 2016;98-B:89-94.

17 Brouwer RW, Jakma TS, Verhagen AP. Braces and orthoses for treating osteoarthritis of the knee. Cochrane Database Syst Rev 2005;1:CD004020.

18 Cudejko T, van der Esch M, van der Leeden M, et al. Effect of soft braces on pain and physical function in patients with knee osteoarthritis: systematic review with meta-analyses. Arch Phys Med Rehabil 2018;99:S390-1.

19 Ouyang J-H, Chang K-H, Hsu W-Y, et al. Non-elastic taping, but not elastic taping, provides benefits for patients with knee osteoarthritis: systemic review and meta-analysis. Clin Rehabil 2018;32:3-17.

20 Lu Z, Li X, Chen R, et al. Kinesio taping improves pain and function in patients with knee osteoarthritis: a meta-analysis of randomized controlled trials. Int J Surg 2018;59:27-35.

21 Reilly KA, Barker KL, Shamley D. A systematic review of lateral wedge orthotics - how useful are they in the management of medial compartment osteoarthritis? Knee 2006;13:177-83.

22 Deveza LA, Loeser RF. Is osteoarthritis one disease or a collection of many? Rheumatology 2018;57:iv34-42.
23 Loeser RF, Goldring SR, Scanzello CR, et al. Osteoarthritis: a disease of the joint as an organ. Arthritis Rheum 2012;64:1697-707.

24 Bierma-Zeinstra SMA, Verhagen AP. Osteoarthritis subpopulations and implications for clinical trial design. Arthritis Res Ther 2011;13:213.

25 Sun X, loannidis JPA, Agoritsas T, et al. How to use a subgroup analysis: users' guide to the medical literature. JAMA 2014;311:405-11.

26 Hancock M, Herbert RD, Maher CG. A guide to interpretation of studies investigating subgroups of responders to physical therapy interventions. Phys Ther 2009;89:698-704.

27 Fisher DJ, Carpenter JR, Morris TP, et al. Meta-analytical methods to identify who benefits most from treatments: daft, deluded, or deft approach? BMJ 2017;356:j573.

28 Kraemer HC. Messages for clinicians: moderators and mediators of treatment outcome in randomized clinical trials. Am J Psychiatry 2016;173:672-9.

29 Stewart LA, Clarke M, Rovers M, et al. Preferred reporting items for systematic review and meta-analyses of individual participant data: the PRISMA-IPD statement. JAMA 2015;313:1657-65.

30 Riley RD, Lambert PC, Abo-Zaid G. Meta-analysis of individual participant data: rationale, conduct, and reporting. BMJ 2010;340:c221.

31 van Middelkoop M, Lohmander S, Bierma-Zeinstra SMA. Sharing data-taming the beast: barriers to meta-analyses of individual patient data (IPD) and solutions. BMJ 2020

32 Lo B. Sharing clinical trial data: maximizing benefits, minimizing risk. JAMA 2015;313:793-4.

33 Packer M. Data sharing in medical research. BMJ 2018;360:k510.

34 The OA Trial Bank. Individual patient data meta-analysis in osteoarthritis research. Available: http://www.oatrialbank.com/home [Accessed 29 May 2020].

35 PROSPERO International prospective register of systematic reviews. Available: http://www.crd.york.ac.uk/prospero

36 Moher D, Shamseer L, Clarke M, et al. Preferred reporting items for systematic review and meta-analysis protocols (PRISMA-P) 2015 statement. Syst Rev 2015;4:1.

37 Shamseer L, Moher D, Clarke M, et al. Preferred reporting items for systematic review and meta-analysis protocols (PRISMA-P) 2015 elaboration and explanation. BMJ 2015;349:g7647.

38 Holden MA, Burke DL, Runhaar J, et al. Subgrouping and targeted exercise pRogrammes for knee and hip osteoarthritis (STEER OA): a systematic review update and individual participant data metaanalysis protocol. BMJ Open 2017;7:e018971.

39 Deveza LA, Bierma-Zeinstra SMA, van Spil WE, et al. Efficacy of bisphosphonates in specific knee osteoarthritis subpopulations: protocol for an oa trial bank systematic review and individual patient data meta-analysis. BMJ Open 2018;8:e023889.

40 van Middelkoop M, Arden NK, Atchia I, et al. The OA trial bank: meta-analysis of individual patient data from knee and hip osteoarthritis trials show that patients with severe pain exhibit greater benefit from intra-articular glucocorticoids. Osteoarthritis Cartilage 2016;24:1143-52.

41 Union EPatCotE. General data protection regulation. Available: https://gdpr-info.eu/ [Accessed 29 May 2020].

42 Covidence systematic review software veritas health innovation, Melbourne, Australia. Available: www.covidence.org

43 Bramer WM, Milic J, Mast F. Reviewing retrieved references for inclusion in systematic reviews using endnote. J Med Libr Assoc 2017; $105: 84$

44 Higgins JPT, Sterne JAC, Savović J, et al. A revised tool for assessing risk of bias in randomized trials.. In: Chander J, McKenzie J, Boutron I, et al, eds. Cochrane database of systematic reviews, 2016.

45 Quartagno M, Carpenter JR. Multiple imputation for IPD metaanalysis: allowing for heterogeneity and studies with missing covariates. Stat Med 2016;35:2938-54.

46 Koopman L, van der Heijden GJMG, Grobbee DE, et al. Comparison of methods of handling missing data in individual patient data metaanalyses: an empirical example on antibiotics in children with acute otitis media. Am J Epidemiol 2008;167:540-5.

47 Riley RD, Lambert PC, Staessen JA, et al. Meta-analysis of continuous outcomes combining individual patient data and aggregate data. Stat Med 2008;27:1870-93.

48 Jüni P, Reichenbach S, Dieppe P. Osteoarthritis: rational approach to treating the individual. Best Pract Res Clin Rheumatol 2006;20:721-40.

49 Zhang S, Paul J, Nantha-Aree M, et al. Empirical comparison of four baseline covariate adjustment methods in analysis of continuous outcomes in randomized controlled trials. Clin Epidemiol 2014;6:227. 
50 Moeller J. A word on standardization in longitudinal studies: don't. Front Psychol 2015;6:1389-93.

51 Higgins J, Thomas J, Chandler J, eds. Cochrane handbook for systematic reviews of interventions version 6.0 (updated July 2019): Cochrane, 2019.

52 Langan D, Higgins JPT, Jackson D, et al. A comparison of heterogeneity variance estimators in simulated random-effects metaanalyses. Res Synth Methods 2019;10:83-98.

53 Fisher DJ. Two-Stage individual participant data meta-analysis and generalized forest plots. Stata J 2015:15:369-96.

54 Higgins JPT, Thompson SG, Deeks JJ, et al. Measuring inconsistency in meta-analyses. BMJ 2003;327:557-60.

55 Higgins JPT, Thompson SG. Quantifying heterogeneity in a metaanalysis. Stat Med 2002;21:1539-58.

56 Lee H, Herbert RD, Lamb SE, et al. Investigating causal mechanisms in randomised controlled trials. Trials 2019;20:1-5.
57 Sterne JAC, Sutton AJ, loannidis JPA, et al. Recommendations for examining and interpreting funnel plot asymmetry in meta-analyses of randomised controlled trials. BMJ 2011;343:d4002.

58 Atkins D, Eccles M, Flottorp S, et al. Systems for grading the quality of evidence and the strength of recommendations I: critical appraisal of existing approaches the grade Working group. BMC Health Serv Res 2004;4:38.

59 Bellamy N, Index WO. A user's guide. London: University of Western Ontario, 1995.

60 Roos EM, Roos HP, Lohmander LS, et al. Knee injury and osteoarthritis outcome score (KOOS)-development of a selfadministered outcome measure. J Orthop Sports Phys Ther 1998;28:88-96.

61 Lequesne MG, Mery C, Samson M, et al. Indexes of severity for osteoarthritis of the hip and knee. Validation--value in comparison with other assessment tests. Scand J Rheumatol Suppl 1987;65:85-9. 